\title{
Accurate energy spectrum for double-well potential: periodic basis
}

\author{
P. Pedram ${ }^{a *}$, M. Mirzaei ${ }^{b}$ and S. S. Gousheh ${ }^{b}$ \\ ${ }^{a}$ Plasma Physics Research Center, Science and Research Branch, Islamic Azad University, Tehran, Iran \\ ${ }^{b}$ Department of Physics, Shahid Beheshti University, Evin, Tehran 19839, Iran
}

June 7, 2010

\begin{abstract}
We present a variational study of employing the trigonometric basis functions satisfying periodic boundary condition for the accurate calculation of eigenvalues and eigenfunctions of quartic double-well oscillators. Contrary to usual Dirichlet boundary condition, imposing periodic boundary condition on the basis functions results in the existence of an inflection point with vanishing curvature in the graph of the energy versus the domain of the variable. We show that this boundary condition results in a higher accuracy in comparison to Dirichlet boundary condition. This is due to the fact that the periodic basis functions are not necessarily forced to vanish at the boundaries and can properly fit themselves to the exact solutions.
\end{abstract}

Keywords: Linear variations; Double-well potential; Eigenvalues; Eigenfunctions; Basis sets.

\section{Introduction}

The phenomena of quantum tunneling in the double-well potential (Fig. 1)

$$
V(x)=-k x^{2}+\lambda x^{4} \quad(k, \lambda>0)
$$

and finding the energy eigenvalues and its stationary states is a long-standing and well-known problem in quantum mechanics. The interest in this problem ranges from various branches of physics to chemistry. After the seminal works by Bender and $\mathrm{Wu}$ [1], numerous papers have been appeared in the literature to tackle this problem. In spite of its simple form, this problem presents a non-trivial model in both quantum mechanics and quantum field theory. In fact, this model can be considered as $0+1$-dimensional quantum field theory $\lambda \phi^{4}$ which has no spatial dimensions.

\footnotetext{
*Email: pouria.pedram@gmail.com
} 
In chemistry, this type of potential corresponds to two equilibrium positions or molecular configurations. The periodic inversion of ammonia $\left(\mathrm{NH}_{3}\right)$ is a well-known example of quantum tunneling. The internal rotation in $\mathrm{CH}_{3} \mathrm{CH}_{3}$ molecule from one configuration to another is a good example of a symmetric double-well potential. On the other hand, the asymmetric $\mathrm{H}_{2} \mathrm{O}_{2}$ hindered rotor or beryllium dicyclopentadienyl represent asymmetric double well potentials. Ammonia $\left(\mathrm{NH}_{3}\right)$, cyanamide $\left(\mathrm{NH}_{2} \mathrm{CN}\right), \mathrm{PH}_{3}$, and $\mathrm{AsH}_{3}$ are chemical examples of symmetrical double well potentials where atoms can tunnel through the barrier.

It is obvious that it is not possible to set up a manageable perturbation theory even for an infinitesimal value of the coupling strength $\lambda$ to calculate the exponentially small energy splitting between quasidegenerate levels in a double-well. This stems from its asymptotic divergent nature [2] due to the eventual dominance of the perturbative correction over the unperturbed contribution for large amplitude of oscillation [1]. This has been the driving force behind the development of non-perturbative methods for this kind of problems. Among them are the semiclassical approximation [3], finite-difference technique [4, hypervirial recurrencerelation scheme [5], renormalized hypervirial-Padè scheme [6], variational matrix solution [7, Rayleigh-Ritz variational method supplemented by the Lanczos algorithm [8], BrillouinWigner perturbation theory based on shifted oscillator variational functions [9, instanton method [10, 11, transfer matrix method [12, uniform approximation of the logarithmic derivative of the ground state eigenfunction [13, and many other specific methods [14, 15, 16, 17]. For the double-well potential, even quite sophisticated techniques, such as the renormalized hypervirial-Padè scheme [], fail to calculate the semi-degenerate low energy states.

The WKB approximation is widely used for its simple mathematical form, but the results are known to be inaccurate due to its inherent defect in the connection formula. One can take the quadratic connection formula instead of those related to the Airy functions to modify the WKB result for the ground state [11, 18. Some other refinements have been developed to improve the accuracy of WKB by changing the phase loss at the classical turning points [19, 20]. The anharmonicity is also taken into consideration in the case of small separation distance between the wells 20$]$. 
In the instanton method, one uses imaginary time path integral to obtain the classical action the socalled Euclidean action. Qualitatively, this method is useful for understanding the quantum tunneling, which has no classical counterpart. However, the quantitative calculation of the energy splitting in the double-well potential by this method is inaccurate because the Euclidean propagator can be obtained only in the limit of infinite separation between the two potential minima [10], which corresponds to zero tunneling probability. Thus, the validity of the instanton approach is restricted to the case in which the two potential minima are far apart, and its accuracy is expected to be reduced as they become close.

Various variational methods are usually used in quantum mechanics, statistical mechanics and field theory. In quantum mechanics, variational parameters are incorporated into trial wave functions and trial Hamiltonians. The Rayleigh-Ritz method is the minimization of the ground state energy with respect to these variational parameters. The applications of this formalism using various set of basis such as harmonic-oscillator 21], Chebyshev polynomials [22], hypervirial theorems [23, and the coherent-states 24, have been already appeared in the literature.

In Refs. [25, 26, 27, 28, it was shown by numerical results that the eigenfunctions which obey Dirichlet boundary condition

$$
\Psi(-L)=\Psi(L)=0
$$

can be effectively used to find the spectrum of an unbounded problem. Then, a critical distance $\hat{L}$ was defined, and it was shown that the low lying energy levels $E_{n}(L)$ are equal to those of $L=\infty$ with high accuracy, if the boundedness parameter $L$ is in near vicinity of $\hat{L}$. In these works, they have employed the Rayleigh-Ritz variational method and have chosen the trigonometric functions (particle-in-a-box) as basis set, in order to solve double-well potential and some other anharmonic symmetric oscillators [25, 26, 27, 28,. They have shown that the trigonometric functions are a suitable basis-set in variational calculations in order to obtain highly accurate results. Moreover, the model is simple, fast-convergent, efficient and works for different kind of potentials $[25,26,27,28$. 
In this paper, we will also use trigonometric basis functions, but with periodic boundary condition

$$
\Psi(-L)=\Psi(L)
$$

In this case, contrary to Dirichlet boundary condition (2), $E_{n}(L)$ has not any relevant minimum value. In fact, for periodic boundary condition $E_{n}(0)$ is zero, whereas $E_{n}(0)$ is infinite for Dirichlet boundary condition. Note that, imposing periodic boundary condition on e.g. the ground state wave function, makes it more accurate than imposing Dirichlet boundary condition. This is due to the fact that the Dirichlet boundary condition enforces the wave function to vanish at $x= \pm L$, but the periodic boundary condition lets the wave function to fit itself more closer to the exact solution which is not necessarily zero at the boundaries. Therefore, we show that using periodic boundary condition, the same accuracy can be obtained with the smaller number of basis.

The paper is organized as follows: In Sec. 2, we outline the general calculation scheme using the periodic boundary condition. In Sec. 3. we apply this method to the case of double-well potential and compare our results with the previous near-exact ones. For the case $k=1$, we find the accurate energy eigenvalues for various value of $\lambda$ and obtain the optimum value of $L$ in each case. Also, we outline the scaling properties of the Hamiltonian to generalize the results to more complicated forms of the potential. In Sec. 4, we state our conclusions.

\section{The variational method}

Let us consider the following dimensionless time-independent one-dimensional Schrödinger equation

$$
\left[-\frac{d^{2}}{d x^{2}}+V(x)\right] \Psi(x)=E \Psi(x)
$$

where $V(x)=V(-x)$. Since the potential is an even function of $x$, to avoid large matrices, we can use even and odd basis functions separately

$$
\phi_{m}^{0}(x)=\frac{1+\delta_{m 0}\left(2^{-1 / 2}-1\right)}{\sqrt{L}} \cos \left(\frac{m \pi x}{L}\right), \quad m=0,1, \ldots
$$




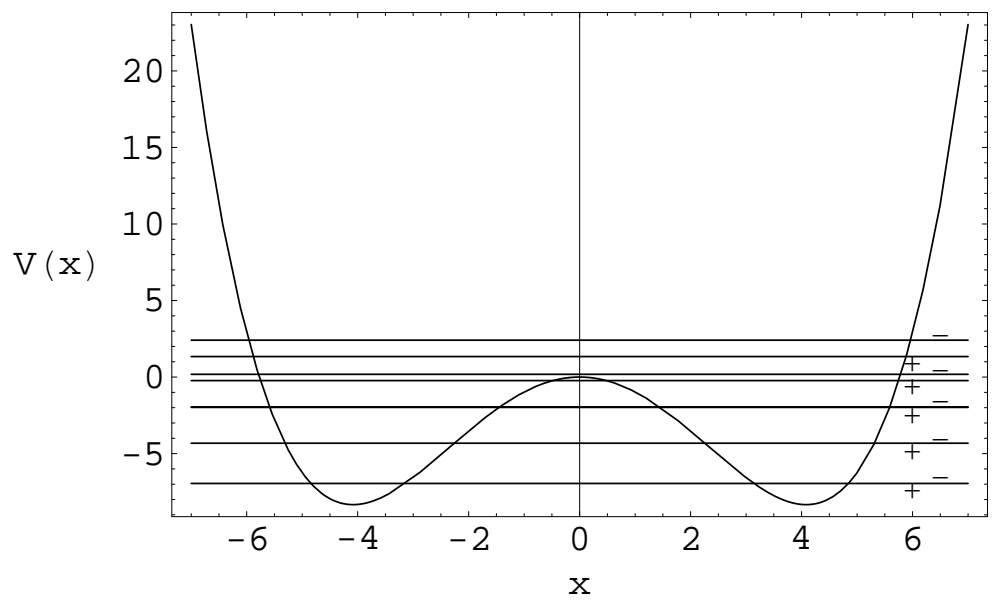

Figure 1: One-dimensional double-well potential where $k=1$ and $\lambda=0.03$. The \pm signs on each energy level refers to the parity of the corresponding eigenstate. Starting from the ground state up, the energy splitting between the levels below the cusp are approximately $\left\{4.3 \times 10^{-6}, 6.1 \times 10^{-4}, 3.0 \times 10^{-2}\right\}$.

and

$$
\phi_{m}^{1}(x)=\frac{1}{\sqrt{L}} \sin \left(\frac{m \pi x}{L}\right), \quad m=1,2, \ldots
$$

Now, we can expand the solution using these orthonormal basis sets

$$
\Psi^{i}(x)=\sum_{m} A_{m} \phi_{m}^{i}(x)
$$

where $i=0,1$ denotes even and odd solutions, respectively. Also, we have the following expansion

$$
V(x) \Psi^{i}(x)=\sum_{m} B_{m} \phi_{m}^{i}(x)
$$

where $B_{m}$ are coefficients that can be determined once $V(x)$ is specified. By substituting Eqs. (77) and (8) into Eq. (4) and using the linear independence of the basis functions, we have

$$
\left(\frac{m \pi}{L}\right)^{2} A_{m}+B_{m}=E A_{m}
$$

Moreover, we can find $B_{m}$ 's using Eq. (8) and (7), namely

$$
B_{m, i}=\sum_{m^{\prime}} C_{m, m^{\prime}} A_{m^{\prime}}
$$

where

$$
C_{m, m^{\prime}}=\int_{-L}^{L} \phi_{m}(x) V(x) \phi_{m^{\prime}}(x) d x
$$


Therefore we can rewrite Eq. (9) as

$$
\left(\frac{m \pi}{L}\right)^{2} A_{m}+\sum_{m^{\prime}} C_{m, m^{\prime}} A_{m^{\prime}}=E A_{m}
$$

By selecting a finite subset of the basis functions, i.e. choosing the first $N$, Eq. (12) can be written as

$$
D A=E A \text {. }
$$

Therefore, the eigenvalues and eigenfunctions of the Schrödinger equation (44) are approximately equal to the corresponding quantities of the matrix $D$.

\section{Results}

To establish the method, first let us study an exactly solvable case where $k=-1$ and $\lambda=0$. Simple Harmonic Oscillator has a well-known energy spectrum $E_{n}=2 n+1$. Figure 2 shows its ground state energy versus $L$ for both periodic and Dirichlet boundary conditions. As apparent from the figure, for the case of periodic boundary condition, the ground state energy starts from zero at $L=0$ and grows as $L$ increases. Moreover, the graph shows the existence of an inflection point around $L=5$ for $N=5$. In this region, the evaluated eigenvalues are close to the exact one $\left(E_{0}=1\right)$. Note that, the position of the inflection point increases for a larger set of basis functions. On the other hand, for Dirichlet boundary condition, the graph of the ground state energy contains a minima around $L=5$ for $N=5$. In this case, the optimal value of $L(\hat{L})$ is the position of this minima on $L$ axis. Also, similar to the previous case, $\hat{L}$ increases for larger values of $N$. This shows that the Rayleigh-Ritz variational formalism is only applicable to the case of Dirichlet boundary condition.

Figure 3 shows the exact and optimized wave functions for $N=1,2$. In fact, for $N \geq 2$ the exact and optimized wave functions are practically indistinguishable on the graph. The optimized ground state

energy for $N=1$ using periodic boundary condition is $1.008\left(E_{0}^{\text {exact }}=1\right)$ whereas this value for Dirichlet boundary condition are 1.136 and 1.006 for $N=1$ and 2, respectively. Therefore, for this case, we need to utilize two oscillatory terms satisfying Dirichlet boundary condition for getting the same accuracy of one oscillatory term which satisfies periodic boundary condition. Moreover, the optimized wave function 
is also more accurate (Fig. 3). This discrepancy is more apparent for larger values of $N$ which will be addressed for the case of a double-well potential. As stated before, this is due to the fact that for the periodic boundary condition, the ground state wave function is not forced to vanish at $x= \pm L$ and can better fit itself to the exact solution which certainly is not zero there.

Now, let us consider the situation that the potential contains an anharmonicity term $(\lambda>0)$. In this case, the sign of $k$ can be either positive (anharmonic oscillator) or negative (double-well potential). In our variational formalism, contrary to Dirichlet boundary condition (2), $E_{n}(L)$ has no minimum value for $L>L_{c}$ (Fig. (4), where $L_{c}$ is the position of classical turning point. Note that, since $\hat{L}$ should be much larger than $L_{c}$ [27, 28], we consider the behavior of $E_{n}(L)$ only for $L>L_{c}$. Figure 4 shows the behavior of the ground state energy for a double-well potential $V(x)=-x^{2}+x^{4}$ and an anharmonic oscillator $V(x)=x^{2}+x^{4}$. As it can be seen from the figure, both plots start from zero and show a semi-flat region for $L>L_{c}$ which contains the sought after inflection point. At this point, the curvature of the curve vanishes. A closer look at the curve shows that this point is close to $L=3.5$ for $N=8, k=1$ and $\lambda=1$. Note that, there also exists a shallow minimum which is due to $L$ being just large enough to detect the minima. Since $L$ is too small in this case to see the overall structure of the potential, this minimum should be ignored. Moreover, the ground state energy of this model is $E_{0} \simeq 0.66$ [26] which is approximately equal to the energy of the inflection point in the left part of Fig. 4 . Therefore, the negative shallow minimum in the figure is not related to the actual value of the energy level which is certainly positive.

To obtain more accurate results, we need to use more basis functions. Table 1 shows the first six lowest energy levels of a double-well potential $(k=1)$ for a small $\lambda$ regime $(\lambda=0.01)$. We have used $N=100$ basis functions in order to obtain the near-exact energy eigenvalues. For this case, the ground state energy is obtained with 82 significant digits which we have only reported it with 30 significant digits. As it is expected, using a fixed $N$, the ground state energy has the maximum precision and the accuracy of the higher energy levels decrease in comparison to the lower ones. To be more precise, the sixth eigenvalue has 79 significant digits which still is very high. Moreover, the optimum length scale 
$\hat{L}$ for 100 basis functions is equal to 16.70762 . This value strictly depends on the number of the basis functions and will increase for a larger set of them. Since the potential shifted by $1 /(4 \lambda)$ is positive definite, some authors have reported the highly accurate positive definite eigenvalues $E_{n}+1 /(4 \lambda)[14$. As it can be seen from Table 1, our results are in complete agreement with them. It is worth to mention that, using the periodic boundary condition, the precision of 30 significant digits can be obtained using 45 basis functions. While, for Dirichlet boundary condition, we need to use 55 basis function to get the same accuracy [26]. In fact, the losing of accuracy is due to the mandatory vanishing of the wave function at $x= \pm L$. Note that, we have also used the optimized value of $L(\hat{L})$ for the ground state energy to compute the higher energy levels $E_{n}$. In fact, we have observed that $\hat{L}$ depends only on the number of basis functions $N$ not on the south after energy levels. This is also true for the case of Dirichlet boundary condition [25, 26, 27, 28]. In principle, we need to use more basis functions to obtain the same accuracy for the higher energy levels. So, reduction of the accuracy of higher energy eigenvalues (using fixed $N$ ) is natural and is not due to the optimization procedure (Tables 1 1 3). Although odd basis functions $\phi_{m}^{1}(x)$ have the same form for both Dirichlet and periodic boundary conditions, their optimized value of $L$ is a little different which results in more accurate eigenvalues even for odd states using periodic boundary condition.

For the larger values of $\lambda$, the accuracy of the results increase even using the same number of basis functions (Table 2 and 3), whereas the optimum value of $L$ decreases. This is due to the decreasing of the classical turning points for larger $\lambda$. These results are also remarkably accurate in comparison with the near-exact values of Refs. [14, 15]. Figure $[5$ shows the optimum value of $L$ versus $N$ for $\lambda=0.01,0.03,0.1$. We can also use the scaling properties of the double-well potential to find the properties of a general Hamiltonian $H(k, \lambda)=-p^{2}-k x^{2}+\lambda x^{4}$ from a more simpler one which we have studied here $H(1, \beta)=-p^{2}-x^{2}+\beta x^{4}$. Using the transformation of variable from $x$ to $k^{1 / 4} x$, we find the following scaling properties $[14,26$,

$$
H(k, \lambda)=k^{1 / 2} H(1, \beta),
$$



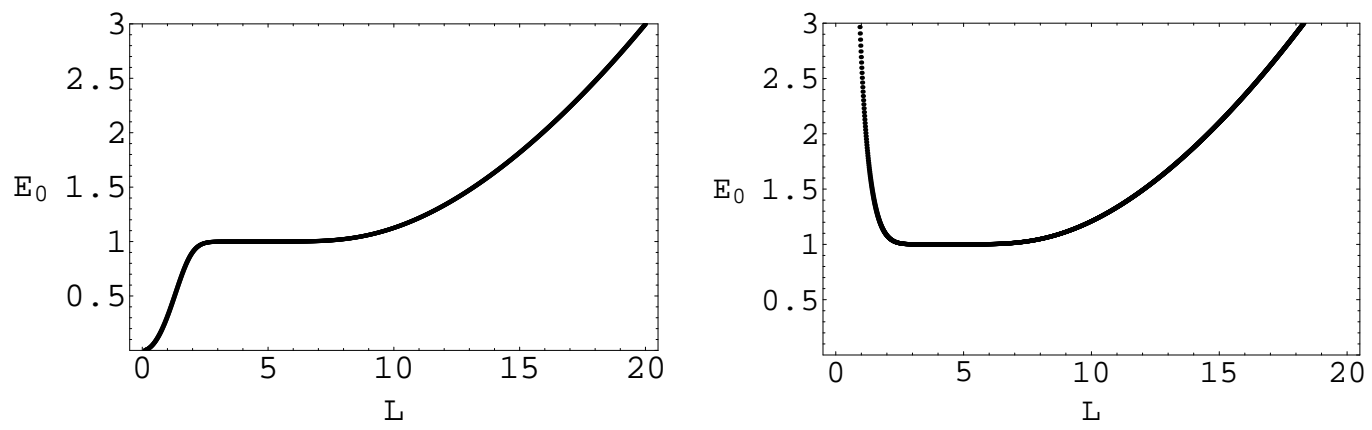

Figure 2: Ground state energy of Simple Harmonic Oscillator versus $L$ for $N=5$ using periodic boundary condition (left) and using Dirichlet boundary condition (right).
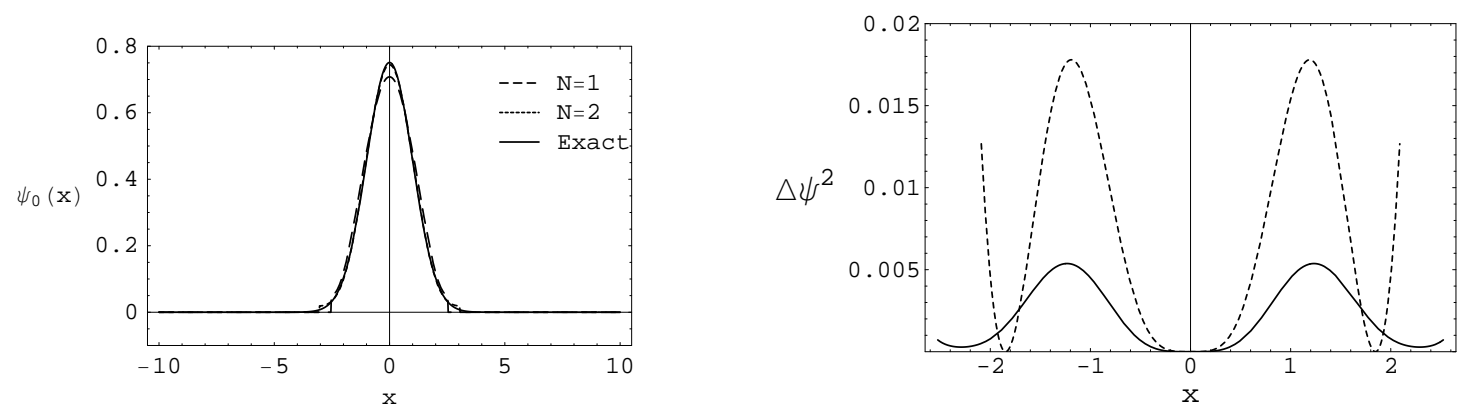

Figure 3: Left, the exact and approximate ground state wave functions of SHO for $\hat{L}(1)=2.52479$ and $\hat{L}(2)=3.04635$. Note that for $N \geq 2$ the exact and approximate wave functions are practically indistinguishable on the graph. Right, $\Delta \Psi_{N=1}^{2}=\left|\Psi_{\text {exact }}-\Psi_{o p}\right|^{2}$ versus $x$ for periodic boundary condition (solid line) and Dirichlet boundary condition (dashed line).

$$
\begin{aligned}
& E(k, \lambda)=k^{1 / 2} E(1, \beta), \\
& \hat{L}(k, \lambda)=k^{-1 / 4} \hat{L}(1, \beta),
\end{aligned}
$$

where $\beta=k^{-3 / 2} \lambda$. Thus, it is sufficient to consider only the reduced potential $(k=1)$.

\section{Conclusions}

We have proposed the usage of periodic boundary condition on the basis function for accurate calculation of eigenvalues and eigenfunctions of a symmetric double-well potential. In this case, the Rayleigh-Ritz variational formalism is not applicable. Because, the graph of the energy versus the domain of the variable starts from zero and grows as this domain increases. We showed that this graph, because of the form of the potential, may contain some minima which are not related to the actual value of the 

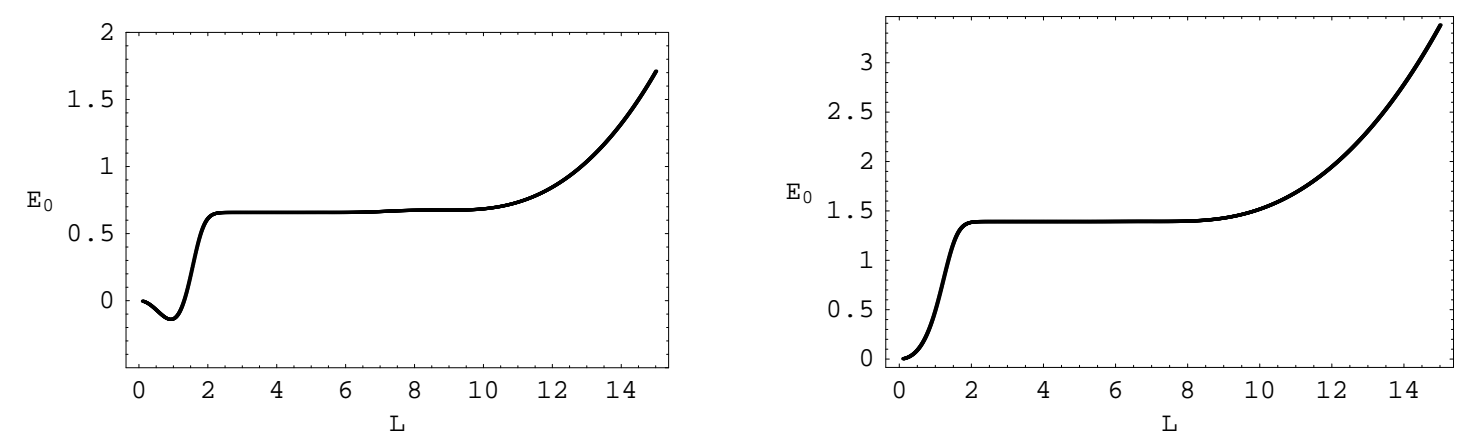

Figure 4: Ground state energy versus $L$ for $N=8, \lambda=1, k=1$ (left), and $k=-1$ (right).

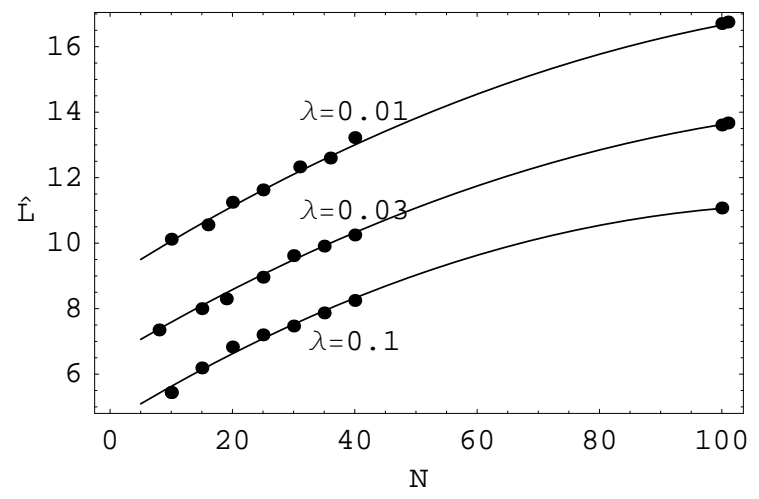

Figure 5: $\hat{L}$ versus $N$ for $k=1$, and $\lambda=\{0.01,0.03,0.1\}$. Circles show the position of the calculated inflection points and the curve is its interpolating function.

Table 1: First six lowest energy eigenvalues of a double-well potential $(k=1)$ for $\lambda=0.01, N=100$ and $\hat{L}=16.70762$. $S D$ denotes the number of significant digits.

\begin{tabular}{ccc}
\hline \hline$n$ & $E_{n}$ & $S D$ \\
\hline 0 & -23.5959513947022931175742924292 & 82 \\
1 & -23.5959513947022931173974337194 & 81 \\
2 & -20.8298063940006898721661249287 & 80 \\
3 & -20.8298063940006897803867088013 & 80 \\
4 & -18.1299111662859753878276848315 & 78 \\
5 & -18.1299111662859531975740043181 & 78 \\
\hline \hline
\end{tabular}

Table 2: First six lowest energy eigenvalues of a double-well potential $(k=1)$ for $\lambda=0.03, N=100$ and $\hat{L}=13.60979$.

\begin{tabular}{ccc}
\hline \hline$n$ & $E_{n}$ & $S D$ \\
\hline 0 & -6.95073188927955191828148104931 & 97 \\
1 & -6.95072754950196756189760500468 & 95 \\
2 & -4.32728413386759375726086836212 & 94 \\
3 & -4.32667786658379381203893295176 & 94 \\
4 & -1.98615994840071249926930230256 & 92 \\
5 & -1.95646376927817057309963393657 & 92 \\
\hline \hline
\end{tabular}


Table 3: First six lowest energy eigenvalues of a double-well potential $(k=1)$ for $\lambda=0.1, N=100$ and $\hat{L}=11.07433$.

\begin{tabular}{ccc}
\hline \hline$n$ & $E_{n}$ & $S D$ \\
\hline 0 & -1.26549283721398510854595401983 & 104 \\
1 & -1.15305913107745006809098709688 & 104 \\
2 & 0.509488545436203212948452569004 & 103 \\
3 & 1.54354603976759862420138901373 & 103 \\
4 & 3.10513379668314777728015050384 & 101 \\
5 & 4.83611381900421025918208666909 & 101 \\
\hline \hline
\end{tabular}

energy and should be ignored. On the other hand, we exhibit that the behavior of the energy versus the variable's domain shows the existence of an inflection point with vanishing curvature which is related to the optimum value of this domain. So, the variational procedure is finding this inflection point by varying the domain of the variable. We also showed that, for a fixed number of basis functions, the results obtained from Dirichlet boundary condition (particle-in-a-box) are less accurate in comparison to the usage of periodic boundary condition. In fact, by using the particle-in-a-box bases, we enforce the wave functions to vanish at the boundaries which results in losing the accuracy. But, the periodic boundary condition lets the wave functions to properly fit themselves to the exact solutions. Therefore, we can get the same accuracy with a smaller number of basis. For instance, for the case of $k=1$, $\lambda=0.01$ and $N=45$ basis functions, we have obtained the energy eigenvalues with 30 significant digits. This accuracy, for Dirichlet boundary condition, is obtainable using $N=55$ particle-in-a-box basis. In fact, this simple and efficient method is rarely used by physicists to tackle eigenvalue problems and even they sometimes use some complicated and inaccurate techniques [29]. Although, we have studied only double-well oscillators, but the presented method is applicable quite generally for eigenvalue problems having polynomial potential which may correspond to physically relevant situations [30]. Moreover, this method can be extended and used for two- or three-dimensional cases. We expect that the priority of the periodic boundary condition to the Dirichlet boundary condition remains also in higher dimensions. In our future work, we will investigate this issue and compare our results with the exact or near-exact ones 31. 


\section{References}

[1] C. M. Bender and T. T. Wu, Phys. Rev. 184, 1231 (1969); Phys. Rev. D 7, 1620 (1973).

[2] L. D. Landau and E. M. Lifschiz, Quantum Mechanics (Butterworth-Heinemann, Oxford, 1998).

[3] S. Y. Slavyanov, Asymptotic Solutions of the One-Dimensional Schrödinger Equation (American Mathematical Society, Providence, 1996); N. Fröman and P. O. Fröman, Phase-Integral Method (Springer-Verlag, New York, 1996); Physical Problems Solved by the Phase-Integral Method (Cambridge University Press, Cambridge, 2002).

[4] M. R. M. Witwit, J. Phys. A 25, 503 (1992).

[5] R. J. Hodgsonand and Y. P. Varshni J. Phys. A 22, 61 (1989); C. R. Handy, Phys. Rev. A 46, 1663 (1992); M. R. Witwitand and J. P. Killingbeck, Can. J. Phys. 71, 475 (1993).

[6] M. Bansal, S. Srivastava, and Vishwamittar, Phys. Lett. A 44, 8012 (1991); S. Srivastava and Vishwamittar, Mol. Phys. 72, 1285 (1991); Mamta and Vishwamittar, Physica A 216, 452 (1995).

[7] R. Balsa, M. Plo, J. Esteveand and A. Pacheco, Phys. Rev. D 28, 1945 (1983).

[8] R. Bishop, M. Flynn, M. Bosca and R. Guardiola, Phys. Rev. A 40, 6154 (1989).

[9] F. Arias de Saavedra and E. Buendia, Phys. Rev. A 42, 5073 (1990).

[10] H. Kleinert, Path Integrals in Quantum Mechanics, Statistics, and Polymer Physics (World Scientific, Singapore, 1990), Chap. 17.

[11] E. Gildener and A. Patrascioiu, Phys. Rev. D 16, 423 (1977); S. Coleman, Phys. Rev. D 15, 2929 (1977); G. Álvarez, J. Math. Phys. 45, 3095 (2004).

[12] Z. Cao, Q. Shen, and F. Zhou, Phys. Rev. A 67, 062112 (2003).

[13] A. Turbiner, Lett. Math. Phys. 74, 169 (2005). 
[14] K. Banerjee and P. Bhatnagar, Phys. Rev. D 18, 4767 (1978).

[15] K. Bandyopadhyay, K. Bhattacharyya and A. K. Bhattacharya, Phys. Lett. A 314, 88 (2003).

[16] V. Fack and G. Vanden Berghe, J. Phys. A 20, 4153 (1987); Pei-Zhu Ding, Xue-Shen Liu, Xiao-Yan Liu, Shou-Fu Pan, Zhong-Yuan Zhou, Int. J. Quantum Chem. 79, 343 (2000).

[17] V. I. Yukalov and E. P. Yukalova, J. Phys. A 29, 6429 (1996); M. Robnik, L. Salasnich, and V. Marko, Nonlinear Phenom. Complex Syst. (Dordrecht, Neth.) 2, 49 (1999); C. S. Park and M. G. Jeong, Phys. Rev. A 58, 3443 (1998); M. A. Jafarizadeh and S. Jalalzadeh, J. Math. Phys. 41, 701 (2000); M. Jafarpour and D. Afshar, J. Phys. A 35, 87 (2002); J. D. Bao and H.-Y. Wang, J. Phys. A 35, 7009 (2002); F. M. Andrade, B. K. Cheng, M. W. Beims, and M. G. E. daLuz, J. Phys. A 36, 227 (2003); M. Battezzati, J. Phys. A 36, 3725 (2003); F. Zhou, Z. Cao, and Q. Shen, Phys. Rev. A 67, 062112 (2003); D. G. Doubropoulos and C. A. Nicolaides, J. Chem. Phys. 119, 8235 (2003).

[18] R. D. Carlitz and D. A. Nicole, Ann. Phys. (N.Y.) 164, 411 (1985).

[19] H. Friedrich and J. Trost, Phys. Rev. Lett. 76, 4869 (1996); H. Friedrich and J. Trost, Phys. Rev. A 54, $1136(1996)$.

[20] M. G. Jeong, C. S. Park, D.K. Park, and S-K. Yoo, Phys. Rev. A 58, 3443 (1998).

[21] J. Killingbeck, Phys. Lett. A 84, 95 (1981); K. Banerjee and J. K. Bhattacharyya, Phys. Rev. D 29, 1111 (1984); R. N. Chaudhuri and M. Mondal, Phys. Rev. A 40, 6080 (1989); R. N. Chaudhuri and M. Mondal, Pramana J. Phys. 37, 13 (1991); F. T. Hioe and E. W. Montroll, J. Math. Phys. 16, 1945 (1975); F. T. Hioe, D. MacMillen and E. W. Montroll, J. Math. Phys. 17, 1320 (1976); F. T. Hioe, D. MacMillen and E. W. Montroll, Phys. Rep. 43, 305 (1978).

[22] J. P. Boyd, J. Math. Phys. 19, 1445 (1978). 
[23] R. J. Swenson and S. H. Danforth, J. Chem. Phys. 57, 1734 (1972); K. Banerjee, Phys. Lett. A 63, 223 (1977); J. Killingbeck, Phys. Lett. A 65, 87 (1978); J. L. Richardson and R. Blakenbecler, Phys. Rev. D 19, 496 (1979); C. S. Lai and M. P. Madan, Mol. Phys. 54, 669 (1985); S. Srivastava and Vishwamitter, Chem. Phys. Lett. 176, 266 (1991).

[24] C. S. Hsue and J. L. Chern, Phys. Rev. D 29, 643 (1984); P. K., Patnaik, Phys. Rev. D 33, 3145 (1986).

[25] H. Taşeli, J. Comput. Phys. 101, 252 (1992).

[26] H. Taşeli, Int. J. Quantum Chem. 46, 319 (1993).

[27] A. Chandra and K. Bhattacharyya, Pramana J. Phys. 43, 117 (1994).

[28] R. Pathak and K. Bhattacharyya, Int. J. Quantum Chem. 54, 13 (1995); R. K. Pathak, A. K. Chandra and K. Bhattacharyya, Phys. Rev. A 48, 4097 (1993); R. K. Pathak, A. K. Chandra and K. Bhattacharyya, Phys. Lett. A 188, 300 (1994); R. K. Pathak and K. Bhattacharyya, Chem. Phys. Lett. 230, 437 (1994); K. Bhattacharyya and R. K. Pathak, Int. J. Quantum Chem. 59, 219 (1996).

[29] G. A. Monerat, E. V. C. Silva, G. Oliveira-Neto, L. G. F. Filho, N. A. Lemos, Phys. Rev. D 73, 044022 (2006); P. Amore, A. Aranda, M. Cervantes, J. L. Dìaz-Cruz, F. M. Fernàndez, Phys. Rev. D 75, 068503 (2007), arXiv:gr-qc/0611029.

[30] P. Pedram, J. Cosmol. Astropart. Phys. 07, 006 (2008), arXiv:0806.1913, P. Pedram, S. Jalalzadeh and S. S. Gousheh, Phys. Lett. B 655, 91 (2007), arXiv:0708.4143, P. Pedram, M. Mirzaei and S. S. Gousheh, Comput. Phys. Commun. 176, 581 (2007), arXiv:math-ph/0701015 S. S. Goushe, H. R. Sepangi, P. Pedram, and M. Mirzaei, Class. Quantum Grav. 24, 4377 (2007), arXiv:gr-qc/0701035 P. Pedram, M. Mirzaei, S. Jalalzadeh, and S. S. Gousheh, Gen. Rel. Grav. 40, 1663 (2008), arXiv:0711.3833, P. Pedram, Int. J. Theor. Phys., 48, 1848 (2009), arXiv:0810.3382, 
[31] H. Taşeli and R. Eid, Int. J. Quantum Chem. 59, 183 (1996). 